\title{
A flexible robotics themed system for teaching basic engineering concepts and skills
}

\author{
Joshua Dian and Sinisa Colic \\ Faculty of Engineering \\ University of Toronto, Toronto, Ontario \\ josh.dian@mail.utoronto.ca
}

\begin{abstract}
The robotics stream of the University of Toronto DEEP summer program consistently attracts numerous motivated high school students. As instructors, we have developed an extensible low cost platform that students can construct and enhance over a sequence of courses. The platform provides an engaging medium to address many aspects of electrical, computer and mechanical engineering while maintaining a robotics theme. These include the theory of electromagnetism, analog and digital circuits, software development and ultimately basic machine learning. Students surveys conducted at the beginning and end of each course were used to gauge student engagement and knowledge before and after the courses and provide evidence that the robotics themed approach is an effective method for teaching a broad spectrum of engineering material.
\end{abstract}

Keywords: Enrichment, Robotics, Laboratory centered

\section{Introduction}

Robotics is a multidisciplinary field encompassing many diverse engineering disciplines. Robotics plays a prominent role in popular culture and provides an ideal method to engage students in science and engineering. Robotic systems take on a variety of forms including familiar "rover" type vehicles and common mechatronic systems such as vending machines. The components of these systems including sensors, decision makers, structural components, and actuators all provide ideal grounds to engage students in experiential learning.
Robotic platforms have been proposed and used for the laboratory component of undergraduate level engineering courses including control theory, mechatronics and social robotics $[4,5,11]$. These systems tend to be complex and are tailored to undergraduate and graduate students with significantly more technical background than high school students. A survey of available robot platforms by Piperidis et al showed that the majority of available robotic platforms for teaching exceed $\$ 750$ in cost leaving few affordable options for teaching high school students [10]. In addition, platforms such as LEGO Mindstorms provide a simple user interface, but limit flexibility and remain expensive. A variety of designs have been proposed which partially address these problems; however, most remain expensive and are not suitable as semi-disposable kits that can be provided to students[2, 6-9].

In this work we propose a simple low cost robotics teaching platform whose construction forms the basis of a variety of laboratory exercises suitable for high school students.

\subsection{DEEP Summer Program}

The DEEP summer academy at the University of Toronto takes place over 4 weeks in July with participants from high schools throughout the world. Courses are targeted toward advanced high school students who apply for and are admitted on a competitive basis. The courses strive to be inclusive of varying students aptitudes and interests while presenting advanced university level material in various subjects. Extracurricular science and engineering programs such as DEEP have been shown to increase student interest and perception of science [3].

The DEEP program provides numerous streams 
of courses including biomedical, environmental and business. This work focuses on the 4 consecutive courses that make up the robotics stream. The robotics stream covers aspects of mechanical design, circuit theory, electromagnetism, embedded software and digital systems as well as artificial intelligence and machine learning. The final course is a design challenge with students given freedom to explore designs within the context of a competition.

The development of the robotic platform has been primarily guided by course teaching experiences to emphasize the following.

- Robotics has broad appeal and as such the systems must be suitable for students of varying knowledge levels and ages. This work is targeted at students in grades 9-12.

- The extracurricular DEEP courses are viewed as "summer camps" by some students making presentation of theoretical and mathematical material challenging. In this light, we use robotics as a method of engaging these students.

- The materials and platform should be low cost such that students can feasibly take home or buy similar materials to continue the courses on their own time.

\subsection{Course Organization}

The courses are organized into modules each comprised of theory and laboratory components focusing on a specific aspect of robotics. In the DEEP program instructors have continuous 5 hour contact with students allowing lectures on theory to be presented in the morning followed by application and laboratory exercises.

Due to the varying ages and experience levels of students, the laboratory exercises are all designed in an open ended fashion. Each exercise culminates in a challenge with levels of increasing difficulty. For example, we begin our navigational challenge with simple linear navigation and progress to complex mazes.

\section{Robotic Platform Overview}

The robotic platform has been developed over the past 5 years as a low cost and simple to construct platform allowing students to rapidly apply theory and concepts presented in lectures.

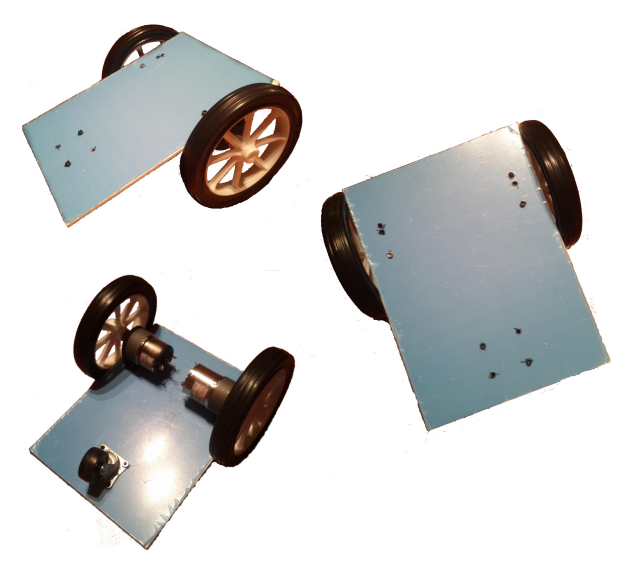

Figure 1: A basic cart constructed by students to be used for the duration of courses.

\subsection{Mechanical Design}

Elements of mechanical design are briefly introduced during initial robot platform construction and covered in more depth during the $4^{t h}$ week. Specifically, students are guided through the design process for a simple robotic cart and for a pick place arm to move objects to different locations. Topics covered include estimation, torque and gearing.

The platform consists of a Plexiglas base plate with two direct current (DC) gear motors and a single rotating caster wheel. Students are able to construct the platform after learning to safely use mechanical workshop tools. A single half day workshop session results in the cart shown in figure 1 . The cart allows a variety of components including sensors, batteries and microcontrollers to be easily added.

\subsection{Electromagnetism and Motors}

Basic electromagnetic theory is presented focusing on providing students with the tools to explain the torque produced in a dc motor and how it results in motion. To reinforce concepts of electromagnetism students construct a simple DC motor and test the motor using standard test equipment. A typical student motor is shown in figure 2. Students are given an outline of the construction procedure but are required to use intuition of magnetic fields and forces from lecture to correctly orient the magnets and coil to produce a functional motor. Finally students use test equipment to measure parameters of the motors attached to their robots. 


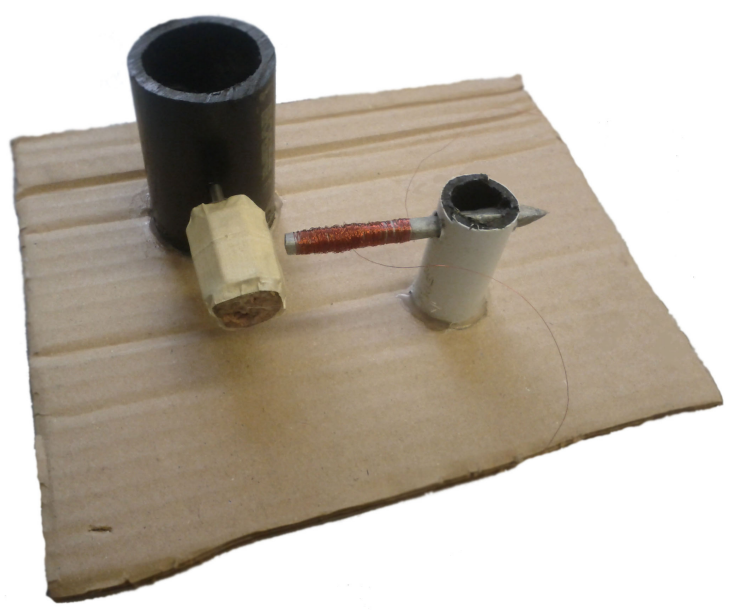

Figure 2: A sample motor constructed by students to illustrate principles of electricity and magnetism.

\subsection{Circuit Theory}

Elements of circuit theory including Kirchhoff's current law (KCL) and Kirchhoff's voltage law (KVL) are presented to students and interspersed with game oriented activities. In a two stage process students are introduced the role of circuit theory in their robot. Students first investigate the accuracy of their calculations in simple resistive circuits. This exercise reinforces the theoretical lesson but also familiarizes students with test equipment and provides an introduction to the debugging process.

Students are finally presented with the design of a low cost DC motor speed controller which is analyzed using the circuit theory techniques presented in the morning. The controller is constructed with basic discrete and circuit parts that can be purchased for less than $\$ 5$. Students subsequently construct the controller and are able to control motor speed and direction.

\subsection{Programming}

The major structures and rules of the C-programming are presented to students in the form of lectures interspersed with brief game like activities. Using the circuit theory skills from previous modules students are guided through the analysis and assembly of an Arduino microcontroller compatible development board [1]. The board produced by students is shown in figure 3 and has been specifically designed to be low cost $(<\$ 7)$, simple and extensible.

The use of simple primatives such as set-

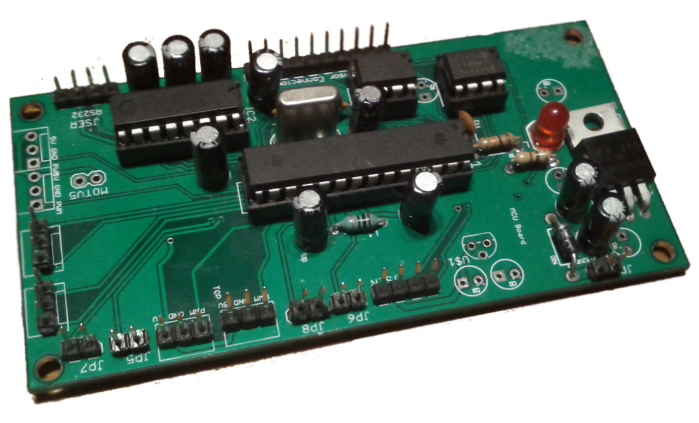

Figure 3: A low cost Arduino compatible robot controller.

Speed(speed1,speed2) where speed 1 and speed 2 are integer values representing forward rotation as positive values and backward rotation as negative values or motors one and two, respectively. Finally students can introduce time delays using the delay(time) primative where time represents a delay in milliseconds. These simple commands allow students very new to programming to accomplish simple navigation tasks as shown in figure 4 .

$$
\begin{aligned}
& \text { //straight } \\
& \text { setSpeed }(10,10) ; \\
& \text { delay }(1000) ; \\
& / / \text { turn } \\
& \text { setSpeed }(10,-10) ; \\
& \ldots
\end{aligned}
$$

Figure 4: Basic C-language primatives used for simple navigation

Students are subsequently presented a series of graded difficulty challenges. The challenges require progressively more complex use of $\mathrm{C}$ programming to control the motion of the robots. This activity provides clear reinforcement for the use of abstract $\mathrm{C}$ language structures and enables students at different aptitude levels to compete in challenges of suitable difficulty.

\subsection{Analog Circuits}

Analog circuits are presented as an extension of circuit theory encompassing operational amplifiers and the basic concepts of frequency domain filter design. The material is again reinforced in two stages. Students are first guided through the characterization of 


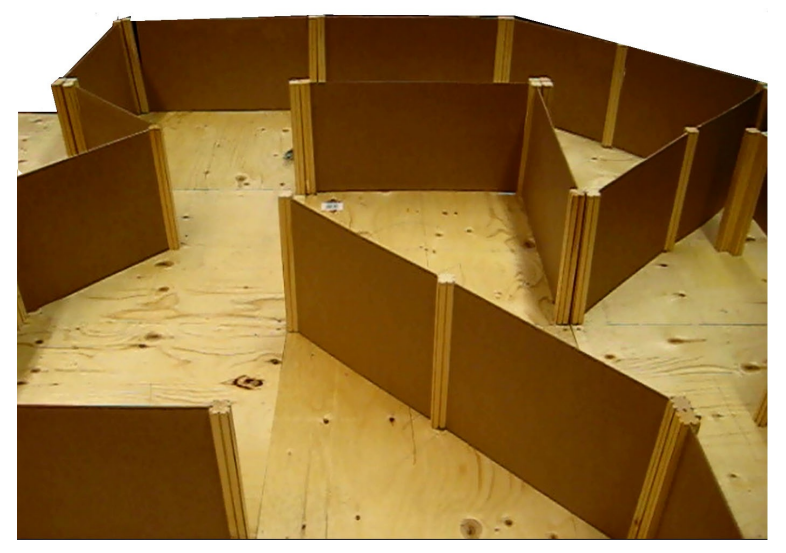

Figure 5: A segment of the maze for robot navigation competitions

a basic operational amplifier circuit using standard test equipment. They subsequently analyze and construct an operational amplifier based circuit appropriate for optical range sensing.

Students are encouraged to characterize their range sensing design and compare the performance to commercially available options. The parts cost in this section is $<\$ 4$ making optical sensors an accessible option for students. Finally, students integrate range sensor into their robot and are able to use $\mathrm{C}$ language structure to enable their robots to perform challenging navigation tasks. A typical maze used for test is depicted in figure 5 and using simple C-language primatives students are able to develop sophisticated navigation strategies.

\section{Results: Student feedback}

Positive anecdotal feedback has been the motivation for continued development of the robotic platform. We have found that students remain engaged and interested longer when activities focused on specific challenges that they could test using robots. Preliminary data obtained from surveys conducted at the beginning and end of the courses suggests that students improved their understanding of major topics presented during the course. Figure 6 depicts the pre and post course survey results for understanding of material related to basic circuits, sensors and actuators.

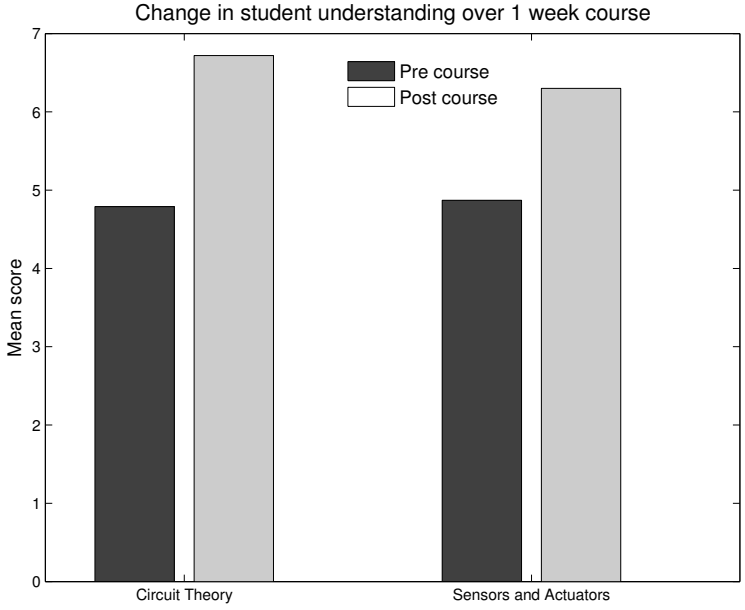

Figure 6: Pre and post course average survey results

\section{Conclusion}

The robotic platform has proven to be a useful method of engaging students and teaching basic concepts of electrical engineering. The platform has been designed to be simple and low cost such that students can purchase and use the platform to continue exploring robotics on their own. Students can easily understand and analyze the design in addition to being able to rapidly construct working versions from commonly available parts. Preliminary survey results suggest that the platform can effectively improve student knowledge of electrical engineering concepts and maintain student interest. Finally we have demonstrated that a robotic platform can be an effective tool for engaging high school students of varied ages and experience levels.

\section{References}

[1] Arduino reference manual, http://arduino . cc/en/Reference/HomePage, Accessed: 201304 .

[2] H. Dinh and T. Inanc, "Low cost mobile robotics experiment with camera and sonar sensors," Proc ACC, St Louis, MO, 2009.

[3] P. Eastwell and L. Rennie, "Using enrichment and extracurricular activities to influence secondary students' interest and participation in science," The Science Educational Review, 2002. 
[4] M. Ferguson, N. Webb, and T. Strzalkowski, "Nelson: a low-cost social robot for research and education," SIGCSE'11, 2011.

[5] G. S. Fischer, W. R. Michalson, T. Padir, and G. Pollice, "Development of a labratory kit for robotics engineering education," AAAI, 2010.

[6] S. Galvan, D. Botturi, A Castellani, and P. Fiorini, "Innovative teaching using lego sets," Proc. IEEE ICRA, Orlando, FL, 2006.

[7] L. Greenwald and J. Kopena, "Mobile robot labs," IEEE Robot. Autom. Mag., vol. 10, no. 2, pp. 25-32, 2003.

[8] J. Hamblen and T. Hall, "Engaging undergraduate students with robotic design projects," Proc. 2nd IEEE DELTA, Perth, Australia, 2004.

[9] J. Lumsden and C. Ortega-Sanchez, "Modular autonomous robotics platform for educational use," Proc IEEE TENCON, Fukoka, Japan., 2010.

[10] S. Piperidis, L. Doitsidis, C. Anastaspoloulos, and N. Tsourveloudis, "A low cost modular robot vehicle design for research and education," Proc. IEEE MED Athens, Greece., 2007.

[11] K. Schilling, H. Roth, and O. J. Rosch, "Mobile mini-robots for engineering education," Global Journal of Engineering Education, vol. 6, no. 1, 2002 . 